\title{
Parathyroid hormone 1-34 inhibits senescence in rat nucleus pulposus cells by activating autophagy via the m-TOR pathway
}

\author{
XIAO-YING WANG ${ }^{1}$, LI-YAN JIAO ${ }^{2}$, JING-LAN HE ${ }^{1}$, ZHI-AN FU $^{1}$ and RU-JUN GUO ${ }^{3}$ \\ ${ }^{1}$ Department of Orthopedic Surgery, The Affiliated Hospital of Hebei University of Engineering; \\ ${ }^{2}$ Department of Dermatology, The Affiliated Hospital of Hebei University of Engineering, Handan, Hebei 056002; \\ ${ }^{3}$ Second Department of Orthopedic Surgery, Wu'an First People's Hospital, Handan, Hebei 056300, P.R. China
}

Received March 9, 2018; Accepted June 20, 2018

DOI: $10.3892 / \mathrm{mmr} .2018 .9229$

\begin{abstract}
Osteoporosis is closely associated with intervertebral disc degeneration. While parathyroid hormone (PTH) 1-34, which is an established drug used to treatosteoporosis, is thought to inhibit the disc degeneration associated with osteoporosis, the precise mechanism involved remains unclear. In the present study, primary Sprague-Dawley rat nucleus pulposus cells (NPCs) were cultured, phenotyped and then treated with dexamethasone (DXM) for $48 \mathrm{~h}$. Cell area analysis and $\beta$-galactosidase staining were used to investigate the effect of DXM on the senescence of NPCs. In addition, the protein levels of LC3-II, Beclin-1, P62, p-mTOR and p-p70S6k were determined by western blotting and analyzing the regulatory effect of PTH upon autophagy and the mTOR signaling pathway in cells treated with DXM. Following autophagic inhibition induced by ATG5 siRNA transfection, the regulatory effect of PTH on senescence in NPCs were investigated in addition to the potential role of autophagy. As the concentration of DXM increased, the size of the NPCs was significantly enlarged and the proportion of cells with positive $\beta$-galactosidase staining increased significantly $(\mathrm{P}<0.05)$. In terms of protein expression, $\mathrm{PTH}$ treatment led to an increase in LC3-II and Beclin-1 proteins, a reduction in $\mathrm{P} 62$ protein, and inhibited p-mTOR and p-p70S6k protein expression in DXM-treated NPCs $(\mathrm{P}<0.05)$. PTH attenuated the effect of DXM according to the cell size and percentage of $\beta$-galactosidase-positive cells. However, the inhibition of autophagy via ATG5 siRNA transfection reversed the protective effect of PTH on cell senescence $(\mathrm{P}<0.05)$. Collectively, the present findings suggest that PTH may inhibit the senescence
\end{abstract}

Correspondence to: Dr Ru-Jun Guo, Second Department of Orthopedic Surgery, Wu'an First People's Hospital, 500 Meters West to KuangJian Road and XiHuan Road Intersection, Handan, Hebei 056300, P.R. China

E-mail: wasyygrj@163.com

Key words: intervertebral disc degeneration, osteoporosis, nucleus pulposus cells, parathyroid hormone 1-34, senescence of NPCs induced by DXM by activating autophagy via the mTOR pathway.

\section{Introduction}

Intervertebral disc degeneration (IDD) is one of the major causes of low back pain (LBP), a condition associated with high morbidity and disability. As a result, LBP has become a significant financial burden worldwide (1). An intervertebral disc consists of three parts: The nucleus pulposus (NP); the annulus fibrosus and the endplate cartilage. The NP is located in the center of the disc and consists of a mixture of nucleus pulposus cells (NPCs) and extracellular matrix, while the outer annulus fibrosus is mainly composed of collagen fibers (2). The NP is the main tissue responsible for axial compression of the spine in the intervertebral disc. As the number of NPCs within a disc decreases and functional capability is lost, the extracellular matrix, which features proteoglycan and type II collagen, becomes degraded. This leads to an increase in the synthesis of type I collagen which ultimately causes discs to degenerate (3). Previous studies have shown that IDD is associated with aging and that spinal disease is an age-related disease. Furthermore, accumulating evidence demonstrates that the senescence of NPCs, as induced by different forms of stress, plays a crucial role in the progression of IDD (4). As NPCs become senescent, their ability to proliferate is impeded and their functional capability is lost (5).

Over recent years, an increasing number of studies have reported that IDD is associated with osteoporosis, although the association remains controversy (6). For example, Luo et al (6) identified a close relationship between IDD and osteoporosis in ovariectomized rats, and speculated that the mechanism involved was related to the integrity and function of adjacent structures of intervertebral discs in the spine. Adjacent segment disc degeneration is very common in women with osteoporosis, thus indicating that osteoporosis might represent one of the causative agents of IDD (7). Research has shown that the endplate of patients with osteoporosis is under high pressure and that this can cause endplate calcification. This structural anomaly exerts influence upon the nutritional infiltration of the end plate, resulting in malfunction of the nutritional supply to intervertebral discs and, ultimately, to IDD (8). 
Parathyroid hormone (PTH) is widely used in the treatment of osteoporosis because it is known to promote the synthesis of bone (9). PTH is also known to regulate the synthesis and metabolism of intervertebral disc cells and inhibit potential calcification molecules via the mitogen-activated protein kinase (MAPK) and protein kinase A (PKA) signaling pathways (9). It has also been reported that PTH cannot only promote anabolic metabolism, but can also inhibit degradation of the extracellular matrix within intervertebral discs (7). Indeed, animal experiments have established that PTH can relieve disc degeneration in rat models of osteoporosis (7). Therefore, PTH has potential clinical significance for the treatment of disc degeneration in patients with osteoporosis, although the exact mechanism involved remains unclear. Previous studies have shown that PTH can promote autophagy in osteoblasts and chondrocytes (10) and can also alleviate osteoarthritis by activating autophagy in articular chondrocytes (11). However, the effect of PTH upon autophagy in NPCs has yet to be evaluated. Dexamethasone (DXM), a type of glucocorticoid, is commonly used in clinical scenarios and is known to cause osteoporosis (12). In the present study, DXM and PTH were used to treat NPCs. We then investigated the levels of senescence and autophagy in order to determine the effect of PTH on NPCs treated with DXM, and also to analyze the potential involvement of autophagy and the mTOR pathway.

\section{Materials and methods}

Ethical approval. The experimental protocol of this study was approved by the Animal Ethics Committee of the Affiliated Hospital of Hebei University of Engineering (Handan, China).

Reagents and antibodies. Alcian dye, rat-derived recombinant PTH and type II collagenase were purchased from Sigma-Aldrich (Merck KGaA, Darmstadt, Germany). Primary antibodies against LC3b, Beclin-1, P62, ATG5, type II collagen, SOX-9 and $\beta$-actin were purchased from Cell Signaling Technology, Inc. (Danvers, MA, USA). $\beta$-galactosidase staining kits, immunofluorescence reagents, western blotting reagents, DAPI, and cell protein extraction kits were purchased from Beyotime Institute of Biotechnology (Shanghai, China). Finally, cell culture reagents, including Dulbecco's modified Eagle's medium (DMEM), fetal bovine serum (FBS) and $0.25 \%$ trypsin were purchased from Gibco (Thermo Fisher Scientific, Inc., Waltham, MA, USA).

Culturing rat primary NPCs. Thirty male Sprague-Dawley rats, aged 3 months and weighing approximately 250-300 g were obtained from SLAC Laboratory Animal Company (Shanghai, China) and treated as described previously (10). In brief, after comforting, the dorsal anatomy was dissected under aseptic conditions and the entire lumbar spine (L1-L6) was dissected and transferred to a clean bench. Each segment of NP (which had a jelly-like appearance) was carefully removed under a microscope and transferred to a centrifuge tube. Collagenase type II was then treated with $0.1 \%$ collagenase for $4 \mathrm{~h}$ at $37^{\circ} \mathrm{C}$ and filtered through a 200 mesh filter. Cells were then cultured in DMEM medium containing $10 \% \mathrm{FBS}$ and $1 \%$ penicillin in an incubator at $37^{\circ} \mathrm{C}$ and $5 \% \mathrm{CO}_{2}$. In order to prevent the dedifferentiation of NPCs, all cells in the follow-up experiments were all second generation.

Experimental design. NPCs were treated with 1,25 and $50 \mu \mathrm{g} / \mathrm{ml}$ of DXM for $48 \mathrm{~h}$. The effect of PTH on autophagy and the mTOR signaling pathway in NPCs was analyzed by dividing cells into four treatment groups: A Control group; DXM group; PTH group; and a DXM + PTH group. All treatments were administered for $48 \mathrm{~h}$. The DXM + PTH group were first treated with $25 \mu \mathrm{g} / \mathrm{ml}$ DXM for $1 \mathrm{~h} ; 10 \mathrm{nM}$ PTH was then added for the remainder of the $48 \mathrm{~h}$ study period. The effect of autophagy on PTH-induced NPC-induced NPC senescence was also analyzed by dividing cells into five treatment groups: A control group; DXM group; DXM + PTH group; DXM + PTH + Control siRNA group; and a DXM + PTH + ATG5 siRNA group. All treatments were administered for $48 \mathrm{~h}$.

Alcian staining. NPCs were plated on 24-well plates at a cell density of $70-80 \%$ and experiments were performed overnight. Cells were fixed with $4 \%$ paraformaldehyde for $10 \mathrm{~min}$ and washed three times with PBS. Then, cells were added with $1 \mathrm{ml}$ of Alcian dye (prepared with acetic acid) and incubated at room temperature for 30 mins. Finally, cells were observed under an inverted microscope (magnification, $\mathrm{x} 10$ ).

Cellular immunofluorescence. Following overnight culture in a 24-well plate, cells were fixed with $4 \%$ paraformaldehyde for $10 \mathrm{~min}$ and then treated with $0.2 \%$ Triton X-100 for $15 \mathrm{~min}$. After blocking with $5 \%$ goat serum for $30 \mathrm{~min}$, cells were incubated overnight at $4^{\circ} \mathrm{C}$ in a refrigerator with primary antibodies against type II collagen and SOX-9 primary (1:100). The following morning, cells were incubated with Alexa Fluor-555 labeled secondary antibody (Beyotime Institute of Biotechnology) for $2 \mathrm{~h}$ at room temperature, stained for $5 \mathrm{~min}$ at room temperature with DAPI (4',6-diamidino-2-phenylindole) (Beyotime Institute of Biotechnology) and finally observed under a fluorescence microscope (Olympus Corporation, Tokyo, Japan).

Measurement of cell surface area. NPCs were treated with different concentrations of DXM for $48 \mathrm{~h}$ and then observed under a microscope and photographed with a 20X objective lens. Then, at least six cells were selected from each image with Image-Pro Plus software (Carlsbad, CA, USA) and measured using the 'Area Measure' function. At least six images from six fields were selected for each treatment group, with at least six cells selected for each image.

$\beta$-galactosidase staining. NPCs were treated with DXM at 25 and $50 \mu \mathrm{g} / \mathrm{ml}$ for $48 \mathrm{~h}$ and then treated with $\beta$-galactosidase (senescence-associated $\beta$-galactosidase, SA- $\beta$-gal) at room temperature for $15 \mathrm{~min}$, in accordance with the manufacturer's instructions. After washing three times with PBS, the cells was stained with SA- $\beta$-gal for $12 \mathrm{~h}$ at room temperature. After washing with PBS, the cells were finally observed under an inverted microscope at $\mathrm{x} 200$, and the proportion (\%) of positive cells present in the total cell population was determined.

Western blotting. Radio-Immunoprecipitation Assay (RIPA, Pikungen, Shanghai), containing $1 \%$ phenylmethanesulfonyl 
fluoride (PMSF) was used to extract total cellular protein and the bicinchoninic acid (BCA) method used to determine protein concentration. Extracted total proteins were then separated by sodium dodecyl sulfate-polyacrylamide gel electrophoresis (SDS-PAGE) and transferred onto polyvinylidene difluoride (PVDF) membranes (Beyotime Institute of Biotechnology) using a wet-spinning method. After blocking with 5\% non-fat milk for $2 \mathrm{~h}$ at room temperature, membranes were washed with TBST for 3 times and then incubated with LC3b, Beclin-1, P62 and ATG5 primary antibodies (1:1,000; Cell Signaling Technology, Inc.) overnight at $4^{\circ} \mathrm{C}$. After 3 washes, the membranes were incubated with horseradish peroxidase-labeled secondary antibody for $2 \mathrm{~h}$ at room temperature. Finally, membranes were treated with ECL luminescence (Thermo Fisher Scientific, Inc.) and positive immune-staining was detected on an imaging system (PerkinElmer, Inc., Waltham, MA, USA). Semi-quantitative analysis of protein bands was performed using AlphaEaseFC 4.0 software (Alpha Innotech Corp., San Leandro, CA, USA).

ATG5 siNRA transcription. The specific design of ATG5 siRNA is shown in Table I (Shanghai GenePharma Co., Ltd., Shanghai, China). $1 \mathrm{ml}$ NPCs with a density of $2 \times 10^{5} / \mathrm{ml}$ were added to 6-well plates and cultured overnight. Cells were transfected using Lipofectamine ${ }^{\circledR} 2000$ (Invitrogen; Thermo Fisher Scientific, Inc.), in accordance with the manufacturer's protocol. At $48 \mathrm{~h}$ after transfection, cells were treated with DXM and PTH as required by the experimental design. The control RNA was a non-specific, non-targeting siRNA. Following transfection, total cellular protein was extracted and analyzed by western blotting.

GFP-LC3 transfection. The GFP-LC3 adenovirus with $10^{10} / \mathrm{ml}$ titer was obtained from Han Heng Biology (Shanghai, China). According to our previous study (13), rat NPCs were cultured on a glass bottom dish overnight and transfected with adenovirus with 100 multiplicity of infection (MOI). According to the $100 \mathrm{MOI}$ and $1-2 \times 10^{6}$ cells in each well, cells were treated with $20 \mu \mathrm{l}$ and $2 \times 10^{8}$ virus, which was diluted into $1 \mathrm{ml}$ serum-free medium. NPCs were cultured with virus for $2 \mathrm{~h}$ in a $37^{\circ} \mathrm{C}$ incubator and then treated with $2 \mathrm{ml}$ normal medium overnight. Then, the transfection efficiency was detected under fluorescence microscopy. Autophagosomes were observed using a laser confocal microscopy (Leica TCS, SP8; Leica Microsystems $\mathrm{GmbH}$, Wetzlar, Germany) after treatments as the experimental design described.

Statistical analysis. All experiments were replicated in triplicate and statistically analyzed by SPSS 15 software package (SPSS, Inc., Chicago, IL, USA). Differences between groups were analyzed by one-way analysis of variance (ANOVA). If ANOVA proved to be statistically significant, then we also used the least significant difference (LSD) method for pairwise analysis. $\mathrm{P}<0.05$ was considered to indicate a statistically significant difference.

\section{Results}

Identification of rat primary NPCs in culture. NP cells were identified as having apolygonal or star-shaped appearance under inverted phase contrast microscopy, and abundant
Table I. Sequences of ATG5 siRNA.

\begin{tabular}{lcc}
\hline Primer & Direction & Sequence 5'-3 \\
\hline ATG5 & Sense & GGCCUUUCAUUCAGAAGCUTT \\
& Antisense & AGCUUCUGAAUGAAAGGCCTT \\
Negative & Sense & UUCUCCGAACGUGUCA CGUTT \\
control & Antisense & ACGUGACACGUUCGGAGAATT
\end{tabular}
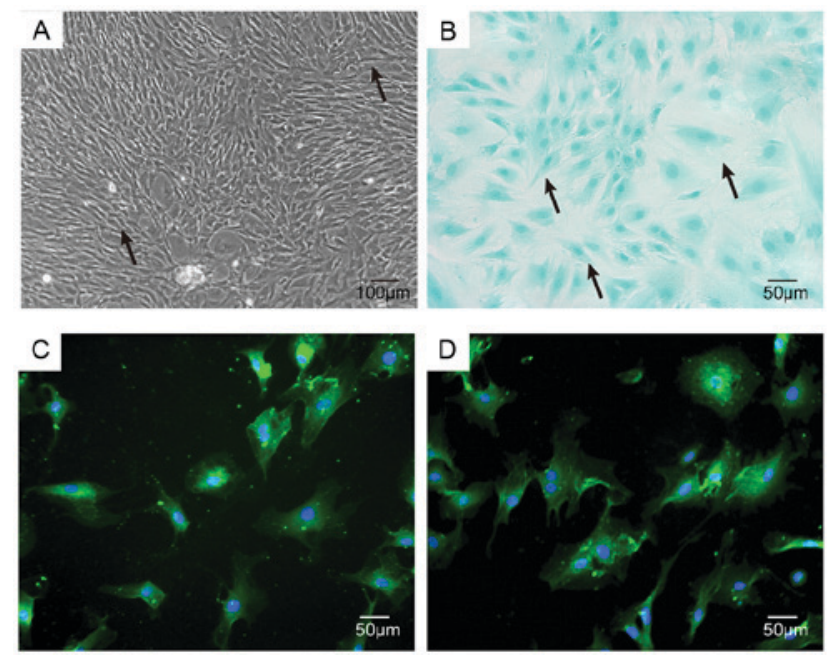

Figure 1. Culture and identification of rat NPCs. (A) NPCs under phase contrast microscopy. The typical cells were indicated by the arrows. (B) Alcian staining. (C) Immunofluorescent staining of Collagen II. (D) Immunofluorescent staining for SOX-9. NPC, nucleus pulposus cell.

particles were observed in the cytoplasm of NPCs (Fig. 1A). Notochord cells containing large number of vacuoles also could be observed. NPCs treated by Alcian staining were light blue in coloration (Fig. 1B) due to the high cellular content of proteoglycan. Further immunofluorescence studies showed that the cytoplasm of NPCs contained large amounts of type II collagen and SOX-9, thus confirming that NPCs exhibit cartilage-like characteristics (Fig. 1C and D).

DXM promoted NPC senescence. After treatment with 1,25 and $50 \mu \mathrm{g} / \mathrm{ml}$ of DXM for $48 \mathrm{~h}$, the cell size of NPCs was significantly increased, particularly in the $50 \mu \mathrm{g} / \mathrm{ml}$ group $(\mathrm{P}<0.05)$. NPC nuclei were enlarged and the cytoplasm was stretched flat, creating a 'fried egg-like' appearance (Fig. 2A). Calculations of cell surface area also demonstrated that glucocorticoid treatment significantly increased the surface area of NPCs $(\mathrm{P}<0.05$; Fig. 2B). The senescence of NPCs was further confirmed by SA- $\beta$-gal staining. The cytoplasm of NPCs treated with 25 and $50 \mu \mathrm{g} / \mathrm{ml}$ DXM were more intensively stained than controls. Semi-quantitative analysis further found that the proportion of NPCs in these two groups that were positively stained with SA- $\beta$-gal was significantly increased $(\mathrm{P}<0.05$; Fig. 2C and D), indicating that NPCs treated with 25 and $50 \mu \mathrm{g} / \mathrm{ml} \mathrm{DXM}$ were senescent.

The effect of PTH on autophagy levels in NPCs. To analyze the effect of PTH on autophagy in NPCs, we used western 
A

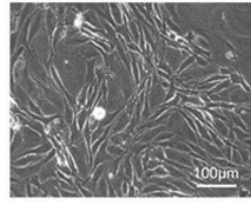

Control

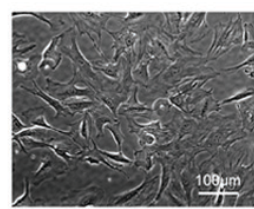

$25 \mu \mathrm{g} / \mathrm{ml}$ DXM

C

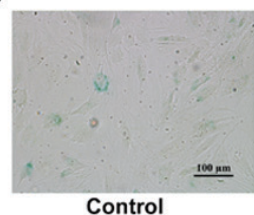

Control

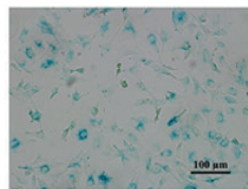

$25 \mu \mathrm{g} / \mathrm{ml} \mathrm{DXM}$

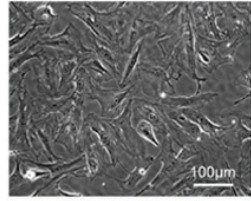

$1 \mu \mathrm{g} / \mathrm{ml} \mathrm{DXM}$

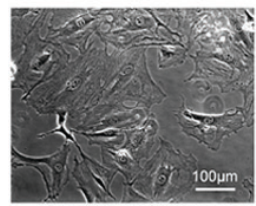

$50 \mu \mathrm{g} / \mathrm{ml}$ DXM

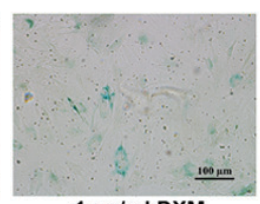

$1 \mu \mathrm{g} / \mathrm{ml}$ DXM

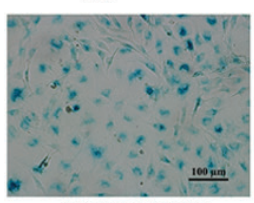

B

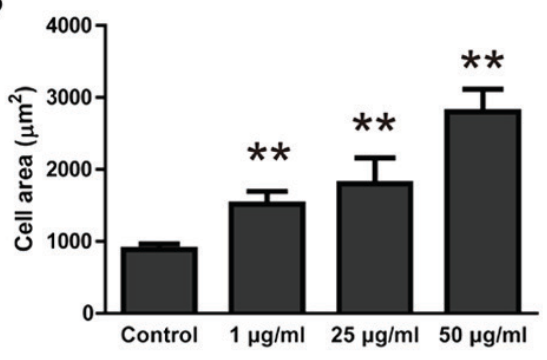

D

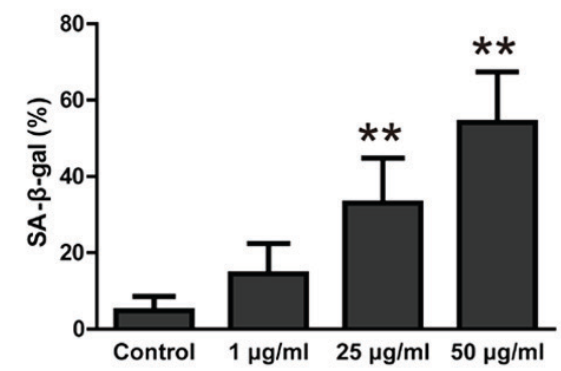

Figure 2. The effect of PTH on senescence in NPCs. (A) Cell morphology under phase-contrast microscopy after DXM treatment. (B) Cell size under phase-contrast microscopy after DXM treatment. ${ }^{* *} \mathrm{P}<0.01$, compared with control groups, $\mathrm{n}=6$. (C) SA- $\beta$-gal staining of NP cells. (D) Semi-quantitative analysis of percentage of SA- $\beta$-gal positive cells. ${ }^{* *} \mathrm{P}<0.01$, compared with control groups, $\mathrm{n}=6$. PTH, parathyroid hormone; NPC, nucleus pulposus cell; DXM, dexamethasone.

blotting and GFP-LC3 assay to analyze the protein expression levels of LC3-II, Beclin-1 and P62, as well as autophagosomes. Compared with the control group, the administration of $10 \mathrm{nM}$ PTH led to a significant increase in the levels of LC3-II protein in NPCs $48 \mathrm{~h}$ later $(\mathrm{P}<0.05)$. The levels of LC3-II in NPCs following DXM + PTH treatment was significantly higher than that of DXM alone $(\mathrm{P}<0.05)$, indicating that PTH cannot only induce NPCs to produceLC3-II protein but can also lead to increased expression of LC3-II in cells treated with DXM $(\mathrm{P}<0.05$; Fig. $3 \mathrm{~A}$ and $\mathrm{B})$. Changes in Beclin-1 levels were similar to those observed with LC3-II ( $\mathrm{P}<0.05$; Fig. 3A and C). Since P62 is a 'transporter' of autophagic degradation-specific proteins, P62 levels gradually decline with increasing PTH concentrations, thus confirming the autophagy of NPCs under PTH-activated DXM ( $\mathrm{P}<0.05$; Fig. 3A and D). Compared with control and DXM group, GFP-LC3 analysis showed that PTH significantly enhanced the number of LC3-positive cells and the number of LC3-positive green dots in one cell (Fig. 3E).

The effect of PTH upon the mTOR signaling pathway in NPCs. mTOR-dependent and mTOR-independent signaling is the main activation mechanism of autophagy, and p70S6K is a signaling molecule downstream of mTOR. The expression of p-mTOR and p-p70S6K in NPCs was significantly reduced after treatment with 10-nM PTH for $48 \mathrm{~h}(\mathrm{P}<0.05)$; a combination of DXM + PTH further reduced the expression of p-mTOR and p-p70S6K compared with $25 \mu \mathrm{g} / \mathrm{ml}$ of DXM alone ( $\mathrm{P}<0.05$; Fig. 4A-D), indicating that PTH can inhibit the mTOR signaling pathway in NPCs exposed to DXM.

Inhibition of autophagy reversed the protective role of PTH on NPC senescence. Next, we transfected NPCs with ATG5 siRNA to suppress autophagy in an effort to analyze the potential involvement of autophagy during the PTH-induced inhibition of senescence in NPCs. Just $48 \mathrm{~h}$ after transfection, Western blotting data showed a significant reduction of ATG5 levels $(\mathrm{P}<0.05$; Fig. 5A and B). Furthermore, ATG5 siRNA significantly inhibited LC3-II levels in NPCs treated with PTH + DXM, demonstrating that ATG5 siRNA resulted in reduced levels of autophagy $(\mathrm{P}<0.05$; Fig. $5 \mathrm{C}$ and $\mathrm{D})$. Compared with DXM alone, a combination of DXM + PTH significantly reduced the surface area of NPCs and reduced the proportion of SA- $\beta$-gal-positive cells, indicating that PTH can inhibit the senescence of NPCs induced by DXM $(\mathrm{P}<0.05$; Fig. 5E and F). However, the transfection of ATG5 siRNA reversed the inhibitory effect of PTH on the surface area of NPCs and proportion of SA- $\beta$-gal-positive cells $(\mathrm{P}<0.05$; Fig. 5E and F). Therefore, autophagy may be involved in the senescent effect of PTH on NPCs.

\section{Discussion}

The treatment of disc degeneration with glucocorticoids is still controversial. One previous study found that injections of glucocorticoid could relieve short-term pain in patients with 

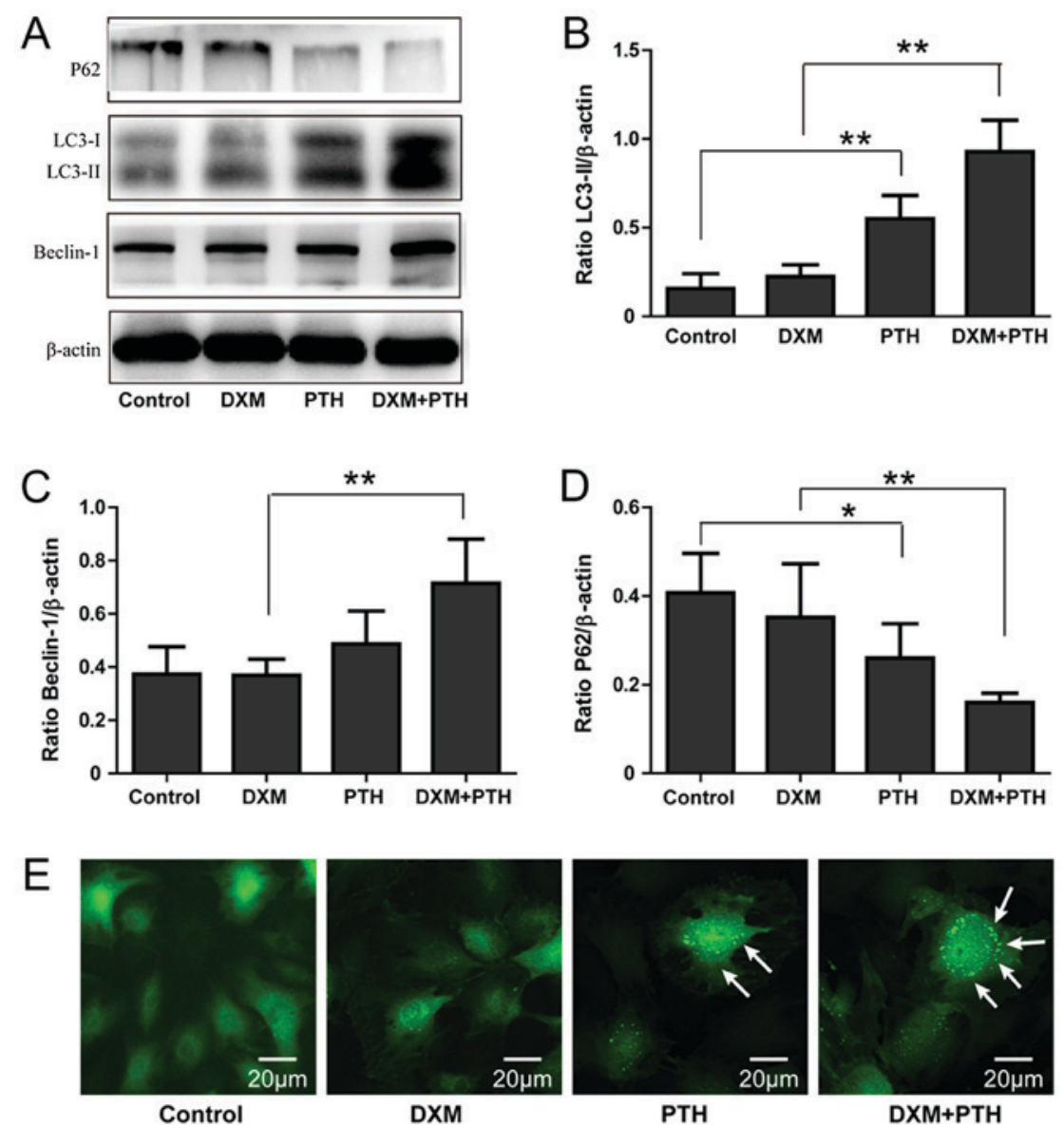

Figure 3. Effect of PTH on the autophagy-related protein and autophagosomes in NPCs treated with $25 \mu \mathrm{g} / \mathrm{ml}$ DXM. (A) Protein levels of LC3-II, Beclin-1 and P62 in NP cells. (B) Semi-quantitative analysis of LC3-II levels. (C) Semi-quantitative analysis of Beclin-1. (D) Semi-quantitative analysis of P62. (E) Autophagosomes in cells treated with DXM and PTH after transfection with GFP-LC3 adenovirus, and the arrow indicated the typical positive GFPL-LC3 dots. ${ }^{*} \mathrm{P}<0.05,{ }^{* *} \mathrm{P}<0.01$, compared between groups, $\mathrm{n}=6$. PTH, parathyroid hormone; NPC, nucleus pulposus cell; DXM, dexamethasone.

\section{A}

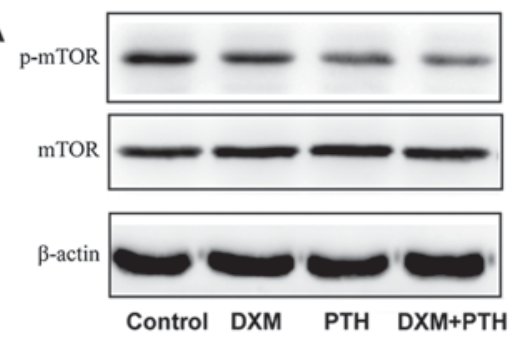

C

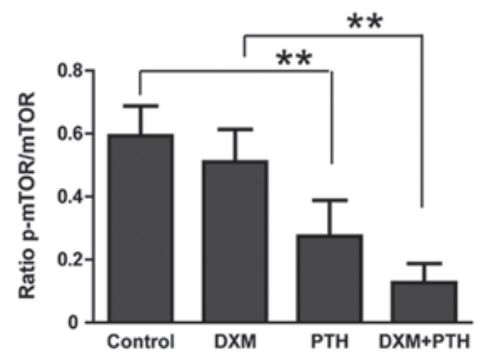

B

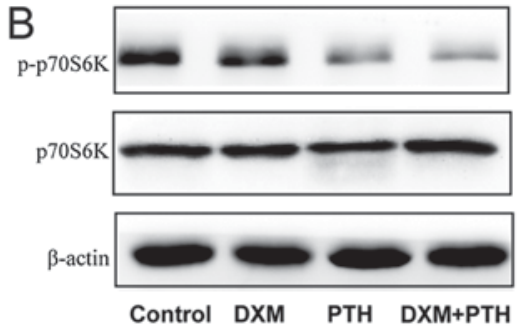

D

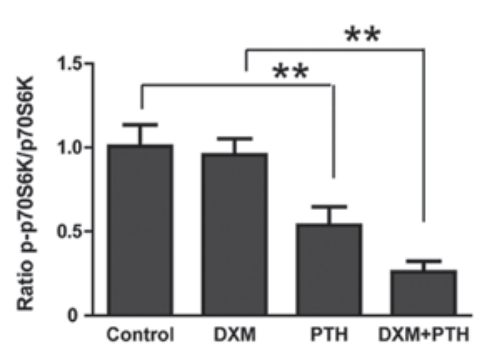

Figure 4. Effect of PTH on p-mTOR and p-p70S6K expression in NPCs treated with $25 \mu \mathrm{g} / \mathrm{ml}$ DXM. (A) Protein levels of p-mTOR as determined by Western blotting. (B) Protein levels of p-p70S6K as determined by western blotting. (C) Semi-quantitative analysis of p-mTOR proteins and (D) Semi-quantitative analysis of p-p70S6K proteins. ${ }^{* *} \mathrm{P}<0.01$, compared between groups, $\mathrm{n}=6$. PTH, parathyroid hormone; NPC, nucleus pulposus cell; DXM, dexamethasone.

acute disc pain, but was not effective over the long-term (11). Although glucocorticoids have the potential to treat IDD as inflammatory reactions, they are also associated with a larger number of complications. For example, Quan et al (14) found that DXM can inhibit the nuclear translocation of the NF- $\mathrm{KB}$ signaling pathway induced by TNF- $\alpha$ and then inhibit 
A

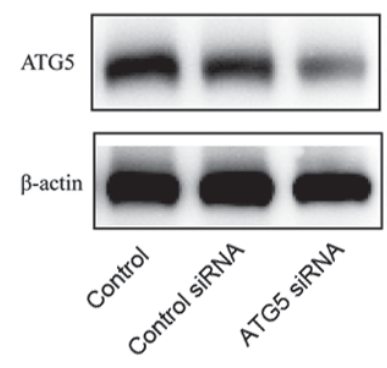

C

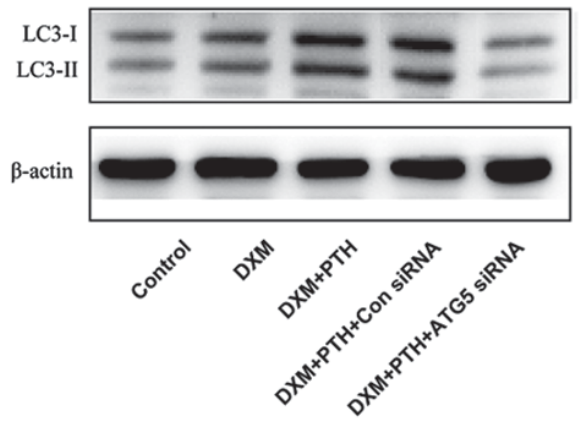

E

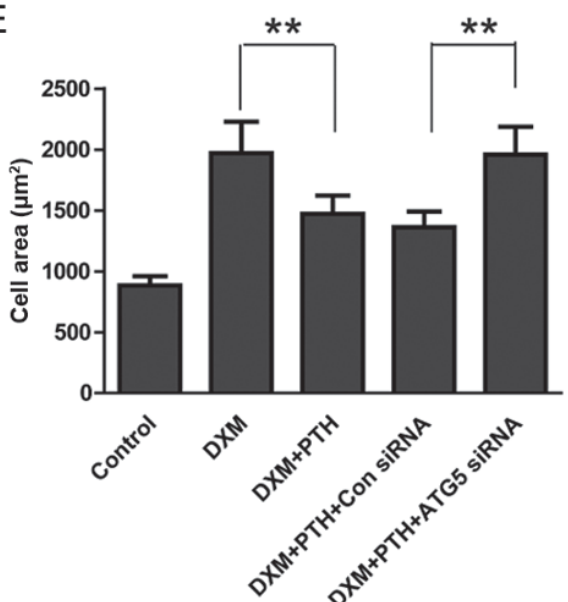

B

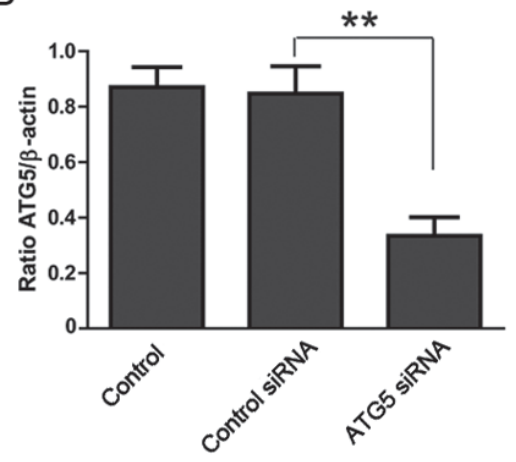

D

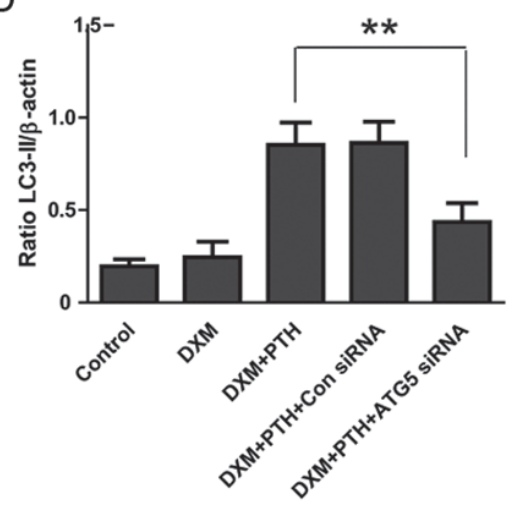

$\mathrm{F}$

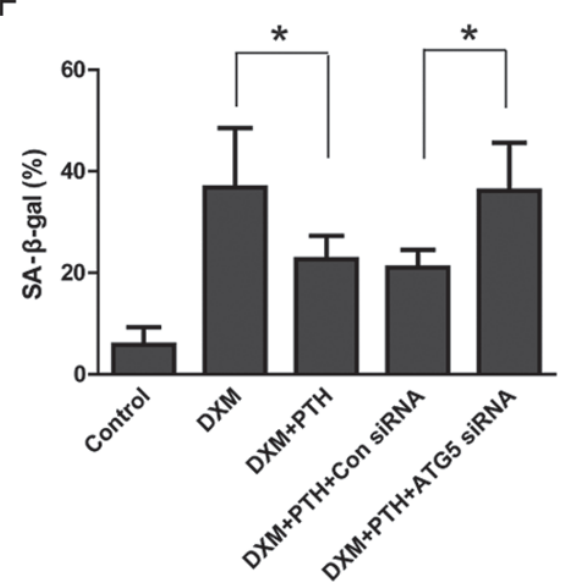

Figure 5. Effect of autophagic inhibition on PTH-induced senescence in NPC cells. (A) ATG5 levels in NPC cells after ATG5 siRNA transfection. (B) Semi-quantitative analysis of ATG5 levels. (C) LC3-II levels after ATG5 silencing. (D) Semi-quantitative analysis of LC3-II levels. (E) Cell size under phase-contrast microscopy. (F) SA- $\beta$-gal staining after ATG5 siRNA transfection. ${ }^{*} \mathrm{P}<0.05,{ }^{* *} \mathrm{P}<0.01$, compared with each group, $\mathrm{n}=6$. PTH, parathyroid hormone; NPC, nucleus pulposus cell.

the inflammatory response of NPCs in a short time period. However, the prolonged use of DXM can stimulate the auto-phosphorylation of NF- $\kappa \mathrm{B}$. The biological characteristics of chondrocyte is similar to that of NPCs. The long-term use of DXM can promote apoptosis and senescence in cartilage cells, prevent the growth of chondrocytes and reduce the functional activity of chondrocytes (15-17). However, the effect of DXM on senescence in NPCs has yet to be reported.

In this study, we found that the cell size of NPCs increased with increasing DXM concentration, and that 25 and $50 \mu \mathrm{g} / \mathrm{ml}$ DMX could significantly increase the proportion of cells that were SA- $\beta$-gal-positive, thus indicating that DXM can promote senescence in NPCs. The mechanism of senescence predominantly involves to pathways: Replicative senescence and precocious senescence. The mechanism underlying replicative senescence is predominantly the p19Arf/p53/p21Cip1/Waf1 signaling pathway, while the mechanism involved in premature senescence is mainly p16INK4a/Rb $(16,17)$; however, the mechanism by which DXM causes senescence in NPCs still needs further study.

Autophagy maintains intracellular homeostasis by clearing damaged organelles and aberrant proteins in cells by fusion with 
lysosomes (18). Autophagy is activated in NPCs when stimulated extracellularly by acid, hypoxia, hypertonicity and starvation. This process leads to the creation of an autophagosome which forms a bilayer membrane structure in the cell. Proteins and organelles, together with lysosomes, also form autolysosomes that degrade damaged organelles and aberrant proteins and thus resist changes in the microenvironment; however, the inhibition of autophagy can accelerate apoptosis in NPCs $(19,20)$. In vivo studies of animals have found that the activation of autophagy can inhibit senescence and apoptosis in NPCs and that IDD can be partially alleviated (21). In the present study, Western blot analysis of the expression of LC3-II, Beclin-1 and P62, established markers of autophagy, and GFP-LC3 assay showed that PTH could activate DXM-induced autophagy in NPCs. Simialrly, Zhu et al (10) reported that $10 \mathrm{nM}$ PTH could promote bone cell autophagy and the inhibition of apoptosis in osteoblast. In another study, Chen et al (11) reported that $0.1 \mathrm{ml} / \mathrm{kg}$ of $10 \mathrm{nM}$ PTH could relieve the progression of osteoarthritis in the knee joint of rats by activating autophagy in chondrocytes. Although DXM has been reported to increase the level of autophagy in chondrocytes and osteocytes (15), the present study indicated that this effect was not likely to be related to the type of cell or the duration of action.

The key molecule underlying the activation of autophagy is the mTOR protein and the mechanisms of involved is usually divided into mTOR-dependent and mTOR independent pathways (22). The activation of mTOR-dependent autophagy involves the inhibition of mTOR expression and a series of processes that cause autophagy in cells after the inhibition of mTOR expression via the downstream mTOR signals p70S6K and 4EBP1 (22). The present study found that PTH inhibits the expression of p-mTOR and p-p70S6K proteins, demonstrating that the mechanism by which PTH activates autophagy may involve an mTOR-dependent signaling pathway. In a previous study, Jiang et al (23) found that high osmotic pressure activated autophagy by inhibiting the mTOR signaling pathway, thus indicating that the mTOR signaling pathway in NPCs might represent the main signaling pathway for the activation of autophagy.

The senescence of NPCs represents one of the mechanisms that causes pathological changes in IDD. Cell senescence blocks the cell cycle, reduces cell viability, increases the expression of catabolic factors and extracellular matrix-degrading enzymes, ultimately leading to changes involving the degradation of extracellular matrix and disc structure (21). Autophagy is closely related to senescence. In human bronchial epithelial cells, Sirt6 activates autophagy via the IGF-Akt-mTOR signaling pathway to suppress cell senescence (24). In NPCs, silencing of mTORC1 by autophagy after mTORC1 activation protects NPCs from inflammation-induced apoptosis, senescence and degradation of the extracellular matrix (25). The present study found that PTH could inhibit DXM-induced senescence in NPCs. However, in cells transfected with ATG5 siRNA, this protective effect was reversed, indicating that autophagy may mediate the inhibitory effect of PTH on senescence in NPCs. Since ATG5 is an important protein in the formation of autophagosomes, ATG5 siRNA is able toinhibit acutophagic activation. The causes of senescence in NPCs often include oxidative stress and inflammatory reactions. However, previous studies have reported that autophagy can inhibit the degree of oxidative stress and inflammation, so this may represent the mechanism underlying the autophagic inhibition of senescence, although this needs to be confirmed by further study.

Collectively our current data showed that DXM at 25 and $50 \mu \mathrm{g} / \mathrm{ml}$ promoted senescence in NPCs. PTH at a concentration of $10 \mathrm{mM}$ increased the level of autophagy in cells treated by DXM and inhibited the mTOR signaling pathway. PTH could therefore alleviate senescence in NPCs under the action of DXM, while silencing ATG5 to inhibit the level of autophagy could reverse the protective effect of PTH. These results suggest that PTH may induce autophagy via the mTOR signaling pathway to inhibit DXM-induced senescence in NPCs and to protect NPCs.

\section{Acknowledgements}

Not applicable.

\section{Funding}

This work was supported by the project of Handan Municipal Science and Technology Bureau (grant no. 1723208067-2).

\section{Availability of data and materials}

All data generated or analyzed during this study are included in this published article.

\section{Authors' contributions}

XYW and LYJ designed and performed the experiments. XYW, LYJ, JLH, ZAF and RJG analyzed the data. XYW, LYJ and JLH prepared all the figures. XYW, JLH and RJG wrote the paper.

\section{Ethics approval and consent to participate}

The present study was conducted in accordance with the ethical guidelines of the 1975 Declaration of Helsinki and was approved by the Committee of the Affiliated Hospital of Hebei University of Engineering. Animal experiments were performed in compliance with the National Institutes of Health Guide for the Care and Use of Laboratory Animals.

\section{Patient consent for publication}

Not applicable.

\section{Competing interests}

The authors declare that they have no competing interests.

\section{References}

1. Rampersaud YR, Bidos A, Fanti C and Perruccio AV: The need for multidimensional stratification of chronic low back pain (LBP). Spine (Phila Pa 1976) 42: E1318-E1325, 2017.

2. Hoy D, Bain C, Williams G, March L, Brooks P, Blyth F, Woolf A, Vos T and Buchbinder R: A systematic review of the global prevalence of low back pain. Arthritis Rheum 64: 2028-2037, 2012.

3. Neidlinger-Wilke C, Galbusera F, Pratsinis H, Mavrogonatou E, Mietsch A, Kletsas D and Wilke HJ: Mechanical loading of the intervertebral disc: From the macroscopic to the cellular level. Eur Spine J 23 (Suppl 3): S333-S343, 2014. 
4. Jiang L, Zhang X, Zheng X, Ru A, Ni X, Wu Y, Tian N, Huang Y, Xue E, Wang X and Xu H: Apoptosis, senescence and autophagy in rat nucleus pulposus cells: Implications for diabetic intervertebral disc degeneration. J Orthop Res 31: 692-702, 2013.

5. Gao C, Ning B, Sang C and Zhang Y: Rapamycin prevents the intervertebral disc degeneration via inhibiting differentiation and senescence of annulus fibrosus cells. Aging (Albany NY) 10 131-143, 2018.

6. Luo Y, Zhang L, Wang WY, Hu QF, Song HP, Su YL and Zhang YZ: Alendronate retards the progression of lumbar intervertebral disc degeneration in ovariectomized rats. Bone 55: 439-448, 2013.

7. Jia H, Ma J, Lv J, Ma X, Xu W, Yang Y, Tian A, Wang Y, Sun L, $\mathrm{Xu} \mathrm{L}$, et al: Oestrogen and parathyroid hormone alleviate lumbar intervertebral disc degeneration in ovariectomized rats and enhance Wnt//-catenin pathway activity. Sci Rep 6: 27521, 2016.

8. Mizrahi J, Silva M, Keaveny T, Edwards W and Hayes W: Finite-element stress analysis of the normal and osteoporotic lumbar vertebral body. Spine (Phila Pa 1976) 18: 2088-2096, 1993.

9. Madiraju P, Gawri R, Wang H, Antoniou J and Mwale F: Mechanism of parathyroid hormone-mediated suppression of calcification markers in human intervertebral disc cells. Eur Cell Mater 25: 268-283, 2013.

10. Zhu L, Chen J, Zhang J, Guo C, Fan W, Wang YM and Yan Z Parathyroid Hormone (PTH) induces autophagy to protect osteocyte cell survival from dexamethasone damage. Med Sci Monit 23: 4034-4040, 2017.

11. Chen CH, Ho ML, Chang LH, Kang L, Lin YS, Lin SY, Wu SC and Chang JK: Parathyroid hormone (1-34) ameliorated knee osteoarthritis in rats via autophagy. J appl physiol (1985) 124: $1177-1185,2018$.

12. Yin H, Wang S, Zhang Y, Wu M, Wang J and Ma Y: Zuogui Pill improves the dexamethasone-induced osteoporosis progression in zebrafish larvae. Biomed Pharmacother 97: 995-999, 2018.

13. He JL, Dong XH, Li ZH, Wang XY, Fu ZA and Shen N Pterostilbene inhibits reactive oxygen species production and apoptosis in primary spinal cord neurons by activating autophagy via the mechanistic target of rapamycin signaling pathway. Mol Med Rep 17: 4406-4414, 2018

14. Quan M, Park SE, Lin Z, Hong MW, Park SY and Kim YY: Steroid treatment can inhibit nuclear localization of members of the NF-kB pathway in human disc cells stimulated with TNF- $\alpha$ Eur J Orthop Surg Traumatol 25 (Suppl 1): S43-S51, 2015.

15. Zaman F, Chrysis D, Huntjens K, Chagin A, Takigawa M, Fadeel B and Sävendahl L: Dexamethasone differentially regulates $\mathrm{Bcl}-2$ family proteins in human proliferative chondrocytes: Role of pro-apoptotic Bid. Toxicol Lett 224: 196-200, 2014.
16. Hong D, Chen HX, Yu HQ, Wang C, Deng HT, Lian QQ and Ge RS: Quantitative proteomic analysis of dexamethasone-induced effects on osteoblast differentiation, proliferation and apoptosis in MC3T3-E1 cells using SILAC. Osteoporos Int 22: 2175-2186, 2011.

17. Xue E, Zhang Y, Song B, Xiao J and Shi Z: Effect of autophagy induced by dexamethasone on senescence in chondrocytes. Mol Med Rep 14: 3037-3044, 2016.

18. Mizushima N, Levine B, Cuervo AM and Klionsky DJ: Autophagy fights disease through cellular self-digestion. Nature 451: 1069-1075, 2008.

19. Chen K, Lv X, Li W, Yu F, Lin J, Ma J and Xiao D: Autophagy is a protective response to the oxidative damage to endplate chondrocytes in intervertebral disc: Implications for the treatment of degenerative lumbar disc. Oxid Med Cell Longev 2017: 4041768 , 2017.

20. Jiang L, Jin Y, Wang H, Jiang Y and Dong J: Glucosamine protects nucleus pulposus cells and induces autophagy via the mTOR-dependent pathway. J Orthop Res 32: 1532-1542, 2014.

21. Chen J, Xie JJ, Jin MY, Gu YT, Wu CC, Guo WJ, Yan YZ, Zhang ZJ, Wang JL, Zhang XL, et al: Sirt6 overexpression suppresses senescence and apoptosis of nucleus pulposus cells by inducing autophagy in a model of intervertebral disc degeneration. Cell Death Dis 9: 56, 2018.

22. Alayev A and Holz MK: mTOR signaling for biological control and cancer. J Cell Physiol 228: 1658-1664, 2013.

23. Jiang LB, Cao L, Yin XF, Yasen M, Yishake M, Dong J and Li XL: Activation of autophagy via Ca-dependent AMPK/mTOR pathway in rat notochordal cells is a cellular adaptation under hyperosmotic stress. Cell Cycle 14: 867-879, 2015.

24. He J, Zhang G, Pang Q, Yu C, Xiong J, Zhu J and Chen F: SIRT6 reduces macrophage foam cell formation by inducing autophagy and cholesterol efflux under ox-LDL condition. FEBS J 284: 1324-1337, 2017

25. Ito M, Yurube T, Kakutani K, Maeno K, Takada T, Terashima Y, Kakiuchi Y, Takeoka Y, Miyazaki S, Kuroda R and Nishida K: Selective interference of mTORC1/RAPTOR protects against human disc cellular apoptosis, senescence and extracellular matrix catabolism with Akt and autophagy induction. Osteoarthritis Cartilage 25: 2134-2146, 2017.

This work is licensed under a Creative Commons Attribution-NonCommercial-NoDerivatives 4.0 International (CC BY-NC-ND 4.0) License. 